\title{
Results of Hygiene Education of Kitchen Cutting Board by using ATP Inspection - Comparison of Vegetable Cutting Board and Meat Cutting Board
}

By Naomi Katayama, Mayumi Hirabayashi, Akemi Ito, Shoko Kondo, Yui Nakayama, Ayari Naka, Natuki Sasaki, Moe Inuzuka \& Takashi Tamura

Nagoya Women's University

Abstract- Since bacteria grow in high temperature and high humidity, bacterial food poisoning frequently occurs from the rainy season to summer. In Japan, the number of food poisoning cases is high from June to October. Maintaining a hygienic environment in the kitchen is very important for preventing food poisoning. In particular, cutting boards on which various foods are places may cause secondary pollution. Therefore, to avoid food poisoning, this study compared the ATP value of the cutting board before and after the hygiene education using the ATP wiping test and investigated the educational effect. Before hygiene education, the inspector conducted an ATP wiping test on the cutting boards for vegetables and meat that washed before and after cooking and notified the cooks of the values. The inspector conducted hygiene education while showing the cook how to clean the cutting board. The cutting board washed with detergent and sponge, rinsed with running water for 30 seconds or more, then this process was repeated twice.

Keywords: gender; ATP wiping test, Cutting board, Hygiene education, double wash.

GJMR-K Classification: NLMC Code: WA 4

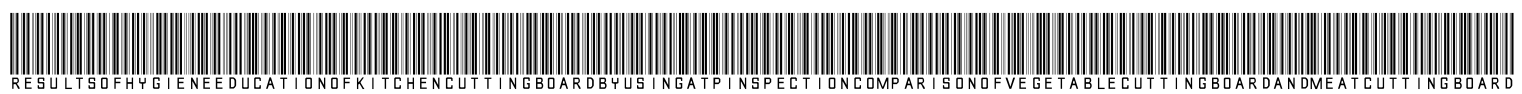

Strictly as per the compliance and regulations of:

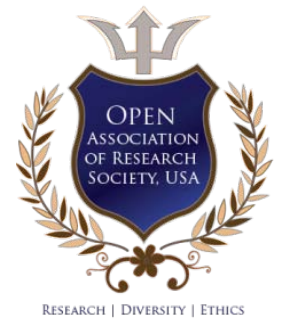

(c) 2020. Naomi Katayama, Mayumi Hirabayashi, Akemi Ito, Shoko Kondo, Yui Nakayama, Ayari Naka, Natuki Sasaki, Moe Inuzuka \& Takashi Tamura. This is a research/review paper, distributed under the terms of the Creative Commons AttributionNoncommercial 3.0 Unported License http://creativecommons.org/licenses/by-nc/3.0/), permitting all non-commercial use, distribution, and reproduction in any medium, provided the original work is properly cited. 


\title{
Results of Hygiene Education of Kitchen Cutting Board by using ATP Inspection - Comparison of Vegetable Cutting Board and Meat Cutting Board
}

\author{
Naomi Katayama ${ }^{\alpha}$, Mayumi Hirabayashi ${ }^{\circ}$, Akemi Ito ${ }^{\circ}$, Shoko Kondo ${ }^{\omega}$, Yui Nakayama ${ }^{*}$, Ayari Naka ${ }^{\S}$,

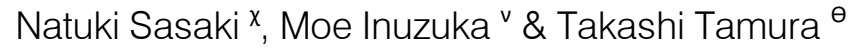

Abstract- Since bacteria grow in high temperature and high humidity, bacterial food poisoning frequently occurs from the rainy season to summer. In Japan, the number of food poisoning cases is high from June to October. Maintaining a hygienic environment in the kitchen is very important for preventing food poisoning. In particular, cutting boards on which various foods are places may cause secondary pollution. Therefore, to avoid food poisoning, this study compared the ATP value of the cutting board before and after the hygiene education using the ATP wiping test and investigated the educational effect. Before hygiene education, the inspector conducted an ATP wiping test on the cutting boards for vegetables and meat that washed before and after cooking and notified the cooks of the values. The inspector conducted hygiene education while showing the cook how to clean the cutting board. The cutting board washed with detergent and sponge, rinsed with running water for 30 seconds or more, then this process was repeated twice. The cooks cooled again, and the inspectors again checked the ATP value on the cutting boards for vegetables and meat that cleaned before and after cooking using the ATP wiping test. As a result, the ATP value of the cutting board before hygiene education was statistically significantly lower than the ATP value by washing after cooking, but it did not fall below 100 . However, after the hygiene education, the ATP value was less than 100 , and it found that the hygiene education affected. It found that hygiene education for preventing food poisoning in the kitchen can effectively performed by making invisible bacteria visible numerically as the ATP value by the ATP wiping test.

Keywords: gender; ATP wiping test, Cutting board, Hygiene education, double wash.

\section{InTRODUCTION}

r n Japan, bacterial food poisoning frequently occurs from the hot and humid rainy season to summer. This season is because bacteria are high temperature and humid and tend to multiply. The number of past food poisoning notifications to the Japanese Ministry of

Corresponding Author a: Nagoya Women's University, Nagoya City, Japan, Graduate School of Nagoya Women's University, Nagoya City, Japan, Department of Otorhinolaryngology, Nagoya University Graduate School of Medicine, Nagoya, Japan.e-mail: naomik@nagoya-wu.ac.jp Author $\sigma \rho$ : Graduate School of Nagoya Women's University, Nagoya City, Japan.

Author $\omega:$ Watanabe Hospital, Mihama town, Noma, Aichi, Japan. Author $¥$ : Nagoya cooking School, Nagoya, Aichi, Japan.

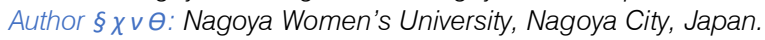

Health, Labor, and Welfare tends to be high from May to October. Alao, these numbers are only those delivered to Public Health Center, so it presumed that they are higher. The number of outbreaks of salmonella, vibrio parahaemolyticus, Escherichia coli, etc., which were the representative bacteria for food poisoning has been decreasing year by year. In contrast, no decrease in bacterial food poisoning due to Campylobacter has been observed, and $60 \%$ or more of the bacterial food poisoning cases have observed. Hygienic handling of food is needed. Furthermore, to prevent crosscontamination, sanitary handling of cooking utensils, especially cutting boards on which various foods placed, must be ensured. However, the problem is that the bacteria are so small that they cannot be seen. Since the microorganisms are invisible, it is not possible to see if the cooking utensils are hygienic just by looking at them during cooking. Hospitals perform ATP wiping tests when performing hygiene management, and use the number of microorganisms as a visible ATP value to help protect the sanitary environment ${ }^{1,2}$. Also, the ATP wiping test can be used in kitchens to help maintain a hygienic environment ${ }^{3,4}$. It has also reported that it is useful to provide hygiene education for staff using the ATP wipe test ${ }^{5}$. Therefore, in this study, we performed an ATP wiping test on cutting boards that are susceptible to secondary contamination from various foods in the kitchen and compared the ATP values before and after hygiene education.

\section{il. Materials and Methods}

\section{a) Kitchen cutting board}

The 12 kitchen vegetable cutting board and 12 kitchen meat cutting board prepared in the kitchen were stored in the sterilization storage the day before the start of cooking.

\section{b) ATP inspection procedure}

Each of the 24 cooks carried a kitchen cutting board for vegetables or meat at the start of their work and bring it to the cooking table. Before the education of hygiene, the work start time depends on the working conditions of the cooks. Still the inspector always performed an ATP inspection before using vegetables or meat with a kitchen cutting board. Then, each cook 
finished the work, washed the kitchen cutting board by himself, and they inspected the ATP inspection by the inspector again. The value of ATP recorded. In the same way, after the education of hygiene, the work start time depends on the working conditions of the cooks. Still the inspector always performed an ATP inspection before using vegetables or meat with a kitchen cutting board. Then, each cook finished the work, washed the kitchen cutting board by himself, and inspected the ATP inspection by the inspector again. The value of ATP recorded.

\section{c) Hygiene education procedure}

\section{i. Cleaning instruction}

While showing the ATP result before the hygiene education to the cook, ATP inspector washes the cutting board firmly with detergent and sponge, rinse with running water for 30 seconds or more. Then, the inspector repeated this process twice. The cook tries to do it as same as the inspector did. Then, the cook tries to do it next cooking. After the education of ATP value was scored.

\section{ii. Statistical processing}

The results obtained were compared using statistical methods. The data was statistically

Table 1: ATP test result of the vegetable cutting board before cleaning instruction

\begin{tabular}{lcc}
\hline & \multicolumn{2}{c}{$\begin{array}{c}\text { before cleaning } \\
\text { instruction }\end{array}$} \\
\cline { 2 - 3 } $\begin{array}{c}\text { Vegitable cutting } \\
\text { board }\end{array}$ & before & after \\
\hline Ctting board 1 & 522 & 219 \\
Ctting board 2 & 1234 & 20 \\
Ctting board 3 & 1447 & 22 \\
Ctting board 4 & 1548 & 30 \\
Ctting board 5 & 1771 & 34 \\
Ctting board 6 & 1154 & 18 \\
Ctting board 7 & 201 & 146 \\
Ctting board 8 & 230 & 194 \\
Ctting board 9 & 516 & 128 \\
Ctting board 10 & 1315 & 216 \\
Ctting board 11 & 1554 & 30 \\
Ctting board 12 & 1941 & 646 \\
\hline Average & 1119.42 & 141.917 \\
Standard deviatic & 602.085 & 178.313 \\
Median & 1274.5 & 81 \\
Maximum & 1941 & 646 \\
Minimum & 201 & 18 \\
\hline \hline
\end{tabular}

b) After hygiene education: Vegetable cutting board and meat cutting board

Tables 3 and 4 show the results of ATP wiping tests on cutting board for vegetables and meat after hygiene education. It can see that the average value of processed, was subjected to an $F$ test to determine whether to use a parametric test or nonparametric test. When there is no difference in the $F$ test, the presence or absence of a significant difference was confirmed using the student-t-test with or without a correspondence. If there was a difference in the $F$ test, the presence or absence of a significant difference was confirmed using the Wilcoxon test with a pair or the Mann-Whitney test without correlation.

\section{RESULTS}

a) Before hygiene education: Vegetable cutting board and meat cutting board

Tables 1 and 2 show the results of ATP wiping tests on cutting board for vegetables and meat before hygiene education. It can see that the average value of the ATP values measured after washing before and after cleaning, this data is significantly lowers the ATP value. However, even after washing, the ATP value did not drop below 100 for both vegetables and meat.

Table 2: ATP test result of the meat cutting board before cleaning instruction

\begin{tabular}{lcc}
\hline \hline & \multicolumn{2}{c}{$\begin{array}{c}\text { before cleaning } \\
\text { instruction }\end{array}$} \\
\cline { 2 - 3 } $\begin{array}{c}\text { Meat cutting } \\
\text { board }\end{array}$ & before & after \\
\hline Ctting board 1 & 798 & 131 \\
Ctting board 2 & 928 & 31 \\
Ctting board 3 & 1091 & 590 \\
Ctting board 4 & 1239 & 617 \\
Ctting board 5 & 1290 & 34 \\
Ctting board 6 & 2613 & 51 \\
Ctting board 7 & 528 & 404 \\
Ctting board 8 & 578 & 379 \\
Ctting board 9 & 682 & 127 \\
Ctting board 10 & 964 & 73 \\
Ctting board 11 & 2220 & 2781 \\
Ctting board 12 & 869 & 281 \\
\hline Average & 1150 & 458.25 \\
Standard deviatio & 642.163 & 761.099 \\
Median & 946 & 206 \\
Maximum & 2613 & 2781 \\
Minimum & 528 & 31 \\
\hline \hline
\end{tabular}

the ATP values measured after washing before and after cleaning, this data is significantly lowers the ATP value. After washing, the ATP value was drop below 100 for both vegetables and meat. Both the cutting boards was very hygienic. 
Table 3: ATP test result of the meat vegetable cutting board after cleaning instruction

\begin{tabular}{lcc}
\hline \hline & \multicolumn{2}{c}{$\begin{array}{c}\text { after cleaning } \\
\text { instruction }\end{array}$} \\
\cline { 2 - 3 } $\begin{array}{c}\text { Vegitable cutting } \\
\text { board }\end{array}$ & before & after \\
\hline Ctting board 1 & 566 & 15 \\
Ctting board 2 & 116 & 239 \\
Ctting board 3 & 1147 & 121 \\
Ctting board 4 & 224 & 60 \\
Ctting board 5 & 1228 & 25 \\
Ctting board 6 & 359 & 75 \\
Ctting board 7 & 1338 & 8 \\
Ctting board 8 & 1323 & 38 \\
Ctting board 9 & 1663 & 60 \\
Ctting board 10 & 1382 & 108 \\
Ctting board 11 & 444 & 158 \\
Ctting board 12 & 165 & 133 \\
\hline Average & 829.583 & 86.6667 \\
Standard deviatio & 565.556 & 68.1767 \\
Median & 856.5 & 67.5 \\
Maximum & 1663 & 239 \\
Minimum & 116 & 8 \\
\hline \hline
\end{tabular}

\section{Statistical Processing Results}

a) Comparison of ATP test values of vegetable and meat cutting boards: before and after education

Before and after hygiene education, the results of the ATP wiping test on vegetable and meat cutting boards statistically compared. The results shown in Tables 5 and 6 . There was a statistically significant
Table 4: ATP test result of the meat cutting board after cleaning instruction

\begin{tabular}{lcc}
\hline & \multicolumn{2}{c}{$\begin{array}{c}\text { after cleaning } \\
\text { instruction }\end{array}$} \\
\cline { 2 - 3 } $\begin{array}{c}\text { Meat cutting } \\
\text { board }\end{array}$ & before & after \\
\hline Ctting board 1 & 282 & 76 \\
Ctting board 2 & 283 & 33 \\
Ctting board 3 & 404 & 6 \\
Ctting board 4 & 1451 & 10 \\
Ctting board 5 & 546 & 29 \\
Ctting board 6 & 565 & 51 \\
Ctting board 7 & 167 & 28 \\
Ctting board 8 & 1573 & 51 \\
Ctting board 9 & 247 & 146 \\
Ctting board 10 & 1527 & 69 \\
Ctting board 11 & 900 & 436 \\
Ctting board 12 & 465 & 89 \\
\hline Average & 700.833 & 85.3333 \\
Standard deviatic & 528.527 & 116.985 \\
Median & 505.5 & 51 \\
Maximum & 1573 & 436 \\
Minimum & 167 & 6 \\
\hline \hline
\end{tabular}

difference in the ATP wiping test values after hygiene education for the cutting board for vegetables and meat. Although there was a statistically significant difference even before hygiene education, the ATP wiping test values for vegetables and meat was not less than 100 , so it can say that hygiene is still insufficient.

Table 5: Statistical comparison results : ATP test results of vegetable cutting board before and after cleaning instruction

\begin{tabular}{|c|c|c|c|c|}
\hline \multirow[t]{2}{*}{ Vegitable cutting board } & \multicolumn{2}{|c|}{ before cleaning instrucion } & \multicolumn{2}{|c|}{ after cleaning instruction } \\
\hline & before cooking & after cooking & before cooking & after cooking \\
\hline Average \pm Standard deviation & $1119.4 \pm 602.1$ & $141.9 \pm 178.3$ & $829.6 \pm 565.6$ & $86.7 \pm 68.2$ \\
\hline F test & \multicolumn{2}{|c|}{$\mathrm{p}=0.0001^{* *}$} & \multicolumn{2}{|c|}{$\mathrm{p}=0.0001^{* *}$} \\
\hline \multicolumn{5}{|l|}{ Student-t test } \\
\hline Wilcoxon test & \multicolumn{2}{|c|}{$p=0.002^{*}$} & \multicolumn{2}{|c|}{$p=0.004 * *$} \\
\hline
\end{tabular}

$*=p<0.05,{ }^{* *}=\mathrm{p}<0.01$

Table 6: Statistical comparison results : ATP test results of meat cutting board before and after cleaning instruction

\begin{tabular}{lcccc}
\hline \hline Meat cutting board & \multicolumn{2}{c}{ before cleaning instrucion } & \multicolumn{2}{c}{ after cleaning instruction } \\
\cline { 2 - 5 } & before cooking & after cooking & before cooking & after cooking \\
\hline Average \pm Standard deviation & $1150.0 \pm 642.2$ & $458.3 \pm 761.1$ & $700.8 \pm 528.5$ & $85.3 \pm 117.0$ \\
F test & $p=0.283$ & $p=0.0001^{* *}$ \\
Student-t test & $p=0.008^{* *}$ & & \\
Wilcoxon test & & & $p=0.002^{* *}$ \\
\hline
\end{tabular}

${ }^{*}=\mathrm{p}<0.05,{ }^{* *}=\mathrm{p}<0.01$ 


\section{Discussion}

The ATP wiping test reveals the ATP value within 1 minute, and it is possible to know the number of invisible bacteria ${ }^{6,7}$. For a reason, it used in facilities such as hospitals that require hygiene management ${ }^{8)}$, this time, focusing on the cutting board of the kitchen. We conducted an ATP wiping test, the ATP values measured before washing and after washing after cooking. Before hygiene education, ATP values for vegetables and meat decreased after washing but did not fall below 100. However, after the hygiene education of washing the cutting board twice, the ATP value was less than 100 when washed, and it was clean. The important thing is that the cutting board is filed with various food material many times a day, so it is necessary to clean it every time. However, since microorganisms are invisible, there is a risk of neglecting cleaning. It is time-consuming to wash twice in busy work, but it is necessary to do it. According to the Japanese Ministry of Health, Labor, and Welfare, the number of food poisoning cases was 1330 in FY2019, the number of patients was 17,282 , of which 3 were fatal cases. The breakdown of the number of patients due to food poisoning by the facility was the top three, with $50.4 \%$ for restaurants $16.0 \%$ for caterers and $11.7 \%$ for business establishments. But the hospitals was $0.6 \%$. Since food poisoning will cause many patients to occur once, it is necessary to pay close attention to hygiene management. Since hygiene education by the ATP wiping test is useful, it is need to carry out regular inspections and call attention.

\section{Vi. Conclusions}

Using the ATP wipe test, the effects of hygiene education were compared by ATP value on the cutting boards, which are likely to cause secondary contamination from various foods in the kitchen. Each of the 24 cooks carried a kitchen cutting board for vegetables or meat at the start of their work and prepare it to the cooking table. The inspector conducted an ATP wipe inspection on the cutting boards for vegetables and meat. The ATP values of the cutting board washed before and after cooking before hygiene education were compared. There was a statistically significant difference even before hygiene education, the ATP wiping test values for vegetables and meat was not less than 100, so it can say that hygiene is still insufficient. The cook learned how to wash the hygienic cutting board twice according to the instructions of the auditor, and cooked again. Then, the inspector again inspected the cutting board. The results, there was a statistically significant difference in the ATP wiping test values after hygiene education for the cutting board for vegetables and meat. After washing, the ATP value was drop below 100 for both vegetables and meat. Both the cutting boards was very hygienic. It found that hygiene education for preventing food poisoning in the kitchen can effectively performed by making invisible bacteria visible numerically as the ATP value by the ATP wiping test.

\section{ACKNOWLEDGMENTS}

We would like to thank all the cooks who participated in this experiment. Also, we would like to thank the inspectors who also performed the ATP inspection.

\section{References Références Referencias}

1. Nante N, Ceriale E, Messina G, Lenzi D, Manzi P. Effectiveness of ATP bioluminescence to assess hospital cleaning: a review. (2017) J Prev. Med. Hyg. 58(2): E177-E183.

2. Amodio E, Dubi C. Use of ATP bioluminescence for assessing $h$ eclealiness of hospital surfaces: a review of the published literature (1990-2012). (2014) J infect Public Health 7(2): 92-98.

3. Aycieck H, Oquz U. Karci K. Comparison of results of ATP bioluminescence and traditional ygiene swabbing methods for the deteminaton of surface cleanliness at a hospital kitchen. (2006). Int J Hyg Environ Heatth. 209(2): 203-206.

4. Osimani A, Garofalo C, Clementi F, Tavoletti S, Aquilanti L. Bioluminescence ATP monitoring for the routine assessment of food contact surface cleanliness in a university canteen. (2014). Int $\mathrm{J}$ Environ Res Public Health 17; 11(10): 10824-10837.

5. Lee JH (2018) An investigation of Factors that influence Hygiene Practices at a small Day Care Center. (2018). J Food Prot. 81(1): 158-164.

6. Stanley PE. A review of bioluminescent STP techniques in papid microbiology. (1989) J Biolumin Chemilumin 4(1): 375-380.

7. Stannard CJ, Gibbs PA. Rapid microbiology: application $s$ of bioluminescence in the food industry - a review. (1986) J Biolumin Chemilumin 1(1): 3-10.

8. Griffith CJ, Coooper RA, Gilmore J, Davies C, Lweis $M$. An evaluation of hospital cleaning refimes and standards. (2000) J Hosp Infect. 45(1): 19-28. 